\title{
Implementing Perioperative Care Pathways; Challenges and Opportunities for the Anaesthetists in Sri Lanka
}

\author{
Thamasi Makuloluwa ${ }^{1 *}$, Vasanthi Pinto ${ }^{2}$ \\ ${ }^{1}$ Professor in Anaesthesia, Department of Surgery, Faculty of Medicine, KotelawalaDefence \\ University, Sri Lanka, ${ }^{2}$ Professor of Anaesthesia \& Intensive Care, Department of Anaesthesia \& \\ Intensive Care, Faculty of Medicine, University of Peradeniya, Sri Lanka
}

The overall burden of diseases that may be cured, palliated or treated with surgical intervention is significant and probably rapidly growing. ${ }^{1}$ In the UK, Around 10 million patients undergo a surgical procedure each year in the NHS, with approximately $15 \%$ of them in the high-risk category needing specialized extra-care to ensure the best possible outcomes after surgery. ${ }^{2}$ Though Sri Lankan figures are not available, an upward trend in patients at higher risk from surgery is evident due to complex surgical procedures performed using advancing techniques, increasing life expectancy associated decline in organ functions, and multiple comorbidities complicating the perioperative period. ${ }^{2}$ Non-communicable Diseases (NCDs) are a major challenge, threatening the well-being of people in Sri Lanka compounded by the increasing proportion of the elderly in the population. ${ }^{3}$

\section{Perioperative Outcomes}

The fitness of surgical patients has a significant influence on morbidity, mortality, and recovery in the perioperative period. Though faster recovery from surgery with no or fewer complications is the norm among healthy individuals, recovery may be long and complicated in terms of unfit high-risk individuals with a significant burden on healthcare resources. Improved fitness through risk assessment and optimization in the perioperative period is viewed as a major step in improving the outcomes of surgery with hastened

*Correspondence: Thamasi Makuloluwa

E mail: ptr makuloluwa@hotmail.com

https://orcid.org/0000-0002-2530-7821

Accepted: 08/03/2021

DOI: http:/doi.org/10.4038/slja.v29i1.8826

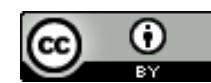

time for recovery and cost benefits to the patient, family, and society. Perioperative Medicine is introduced as an additional level of care from contemplation of surgery until the full recovery to improve outcomes among high-risk categories of patients. ${ }^{2}$

\section{Perioperative Care}

Perioperative care traditionally focuses on surgical outcomes and is mostly limited to the immediate perioperative period with no emphasis on long-term survival and outcomes. However, the novel perioperative care pathways recommend a patient-centered, multidisciplinary, integrated, and organized approach adopted across primary, social, and secondary care settings, until complete recovery from surgery. ${ }^{2,4}$ It consists of preoperative risk assessment and optimization of co-morbidities; lifestyle modification to improve both surgical and long-term health outcomes (prehabilitation); safe and quality intraoperative care and surgery; measures to minimize short and long term effects of surgery and acute illnesses through follow up (rehabilitation), implemented through a 'perioperative care team' ${ }^{2,4}$ Perioperative care pathways aim to deliver safe and quality anaesthetic for all patients, including high-risk patients with high-quality recovery and outcomes. However, the existing perioperative care services do not fall totally in line with the current recommendations and lack the formalized team delivery of holistic, individualized care across the perioperative period for all surgical patients.

\section{Role of Anaesthetists in Perioperative Care: Current Context}

Perioperative care is not a novel concept for anaesthetists. Traditionally, the anaesthetists take overall care of the delivery of immediate perioperative services tailored to the medical needs of individual patients with advice from experts in the field where needed. They play pivotal roles in assessing risk and optimizing the 
general health of patients attending preoperative assessment clinics or those awaiting emergency surgical procedures; by ensuring safe anaesthetics intraoperatively, and continuing perioperative care into high-dependency or intensive care where necessary.

\section{Role of Anaesthetists in Perioperative Care: Future Perspectives}

In recent years, the specialty of anaesthesia has focused on evolving the role of the anaesthetist into that of the 'perioperative physician'. ${ }^{2}$ Considering the expertise of anaesthetists in improving fitness for surgery in the perioperative period, it is observed that they best suit to take up a leading role in the 'perioperative care team' to coordinate a 'continuum of care' at different levels of the perioperative care pathway through integrated multi-disciplinary inputs. They may take the lead in developing standardized perioperative care pathways for all patients based on their medical or surgical risk levels while strengthening risk assessment and optimization through anaesthetist-led clinics and prehabilitation. Being experts in perioperative care, measures can be taken to ensure safety and quality of care early and late after surgery by establishing outreach care, pain services, enhanced recovery programs (ERAS) and expanding facilities in the highdependency and intensive care. ${ }^{4}$

\section{Implementing Perioperative Care Pathways: Challenges and Opportunities for the Anaesthetists}

Executing perioperative care pathways in Sri Lanka will be a challenge. It requires the development of new care models adapted to local settings, with redefined clinical roles for stakeholders in the health care system. ${ }^{2}$ Compared to HICs, implementing such models in LMICs will be more challenging due to limitations in healthcare systems, skilled manpower and other related resources. Under organized primary healthcare system in Sri Lanka with no coordinated referral system from secondary or tertiary care for prehabilitation and rehabilitation is foreseen as a major constraint. Considerable shortage of anaesthetists at all levels is another key limitation in implementing fully-fledged perioperative care pathways in Sri Lanka.

\section{Opportunities}

Despite the challenges, the anaesthetists may take the initiative to establish perioperative care pathways with a view to improving perioperative outcomes. As preliminary measures, assessing and categorizing risk levels of all patients, developing standards for optimization of different risk categories and establishing protocols to facilitate prehabilitation through shared-decision making are essential. Similarly, while ensuring the safety and quality of anaesthetic services, implementing ERAS, strengthening rehabilitation for improving early and late surgical outcomes are other areas that require focus in achieving the expected goals of perioperative care.

Establishing fully-fledged perioperative care bundles in LMICs may take longer. Yet, initiatives should be taken to strengthen the existing perioperative care services by adopting an integrated multidisciplinary team approach and inculcating shared decision-making with patients. As preliminary measures, pathways for prehabilitation and rehabilitation and programs for enhanced recovery for selected high risk surgical patients can be developed and adopted.

\section{References}

1. Bulletin of the World Health Organization; Volume 86, Number 8, August 2008, 577-656

2. Perioperative Medicine - The pathway to better surgical care (RCoA) (perioperativemedicine@rcoa.ac.uk)

3. Prevention and Control of Non-communicable Diseases (2018): Think Globally - Act Locally; Lessons from Sri Lanka. Ministry of Health, Nutrition and Indigenous Medicine, Sri Lanka. ISBN 978- 955 -3666-20 - 8

4. The Centre for Perioperative Care (cpoc.org.uk)

5. Perioperative care in adults; NICE guideline [NG180]; 19 August 2020 